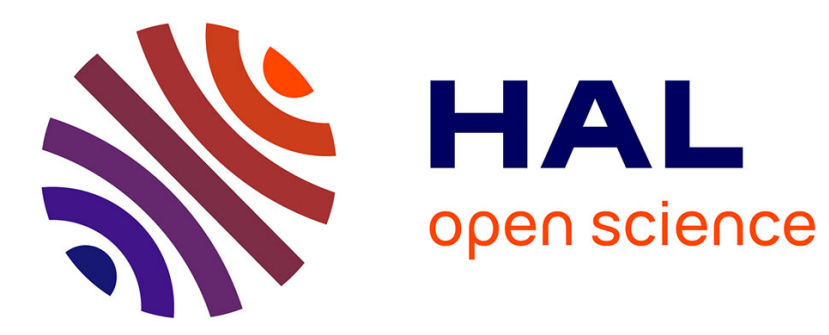

\title{
Towards realistic simulations of sound radiation by moving sources in outdoor environments
}

Didier Dragna, Philippe Blanc-Benon

\section{To cite this version:}

Didier Dragna, Philippe Blanc-Benon. Towards realistic simulations of sound radiation by moving sources in outdoor environments. International Journal of Aeroacoustics, 2014, 13 (5-6), pp.405-426. 10.1260/1475-472X.13.5-6.405 . hal-01136603

\section{HAL Id: hal-01136603 https://hal.science/hal-01136603}

Submitted on 11 Apr 2016

HAL is a multi-disciplinary open access archive for the deposit and dissemination of scientific research documents, whether they are published or not. The documents may come from teaching and research institutions in France or abroad, or from public or private research centers.
L'archive ouverte pluridisciplinaire HAL, est destinée au dépôt et à la diffusion de documents scientifiques de niveau recherche, publiés ou non, émanant des établissements d'enseignement et de recherche français ou étrangers, des laboratoires publics ou privés. 


\title{
Towards realistic simulations of sound radiation by moving sources in outdoor environments
}

\author{
Didier Dragna* and Philippe Blanc-Benon \\ Laboratoire de Mécanique des Fluides et d'Acoustique, UMR CNRS 5509, \\ École Centrale de Lyon, Université de Lyon, \\ 36, avenue Guy de Collongue, 69134 Ecully Cedex, France
}

Submitted: Oct 10, 2013; Revised Apr 30, 2014; Accepted Aug 19, 2014

\begin{abstract}
PACS numbers:43.28.Js, 43.28.En, 43.20.El

A time-domain solver of the linearized Euler equations is used to study outdoor propagation of acoustic waves generated by broadband moving sources. For that, high-order schemes, developed initially in the computational aeroacoustics community, are employed. A time-domain impedance boundary condition recently proposed in the literature is implemented to deal with reflexion of acoustic waves over the ground. In addition, curvilinear coordinates are used to account for topographic effects. First, test cases show that long range sound propagation and diffraction by obstacles in three-dimensional geometries are accurately determined. Simulation of the acoustic radiation by a broadband monopole source moving above a perfectly reflecting plane is then considered. Numerical results are satisfactorily compared to those obtained from an analytical solution. At last, the case of a broadband source moving above a non-flat terrain, with an inhomogeneous impedance ground, is investigated. The effects of a topography defect on the acoustic field are examined.
\end{abstract}

\section{INTRODUCTION}

Increase of transportation speed and traffic has a large impact on populations. Indeed, more than 100 million Europeans are exposed to traffic noise levels which are detrimental to health [1]. Therefore, there is a considerable pressure from policy makers to reduce noise pollution. Industry can then be expected to be faced with more and more drastic regulations in the coming years. As a consequence, there is an urgent need to both reduce noise sources and better predict the propagation of the generated noise in the environment.

However, prediction of noise due to sources in motion outdoors is still an open challenge. Indeed, analytical solutions are only known in very simple cases. The

\footnotetext{
*Corresponding author: E-mail: didier.dragna@ec-lyon.fr
} 
most common problem is concerned with sound radiation by a monopole moving at a constant speed and at a constant height above a flat ground [2, 3, 4]. Analytical solutions have been obtained under strong hypothesis, assuming that the ground properties are constant with frequency $[2,4]$ or neglecting the contributions due to the singularities of the reflection coefficient $[2,3]$. Recently, analytical solutions for the same problem have been proposed without these assumptions by Ochmann [5] but only for simple impedance models. For more general configurations, there are only heuristic approaches [4]. Moreover, existing numerical tools to predict transportation noise such as railway noise or aircraft flyover noise (see e.g. [6, 7, 8]) are based on simple propagation models, such as ray-tracing or parabolic equation methods, which do not account or only partially account for meteorological and ground effects.

As acoustic sources motion is time dependent, time-domain simulations of oudoor sound propagation are therefore well-suited. They have been used for more than ten years especially to study the influence of meteorological conditions on the acoustic field $[9,10,11,12,13,14]$. The objective of the paper is to develop a numerical solver able to account for sources in motion in complex environments. For that, high-order numerical schemes are employed. Topography and reflexion on an impedance ground are accounted for by using curvilinear coordinates and a time-domain impedance boundary condition, respectively.

The paper is organized as follows. In Sec. II, the numerical solver is presented. Two test cases are performed for validation. In Sec. III, the solver is used to simulate the radiation by sources in motion. First, the case of a source moving at a constant speed and at a constant height above a perfectly reflecting plane is considered. Then, the radiation by a moving source above a non-flat terrain with an inhomogeneous ground is examined.

\section{NUMERICAL SOLVER}

\section{A. Equations in curvilinear coordinates}

Different sets of equations can be proposed to describe sound propagation in the atmosphere. For applications in transportation noise, in which the acoustic field does not perturbate the mean flow, it is appropriate to solve the linearized Euler equations. In this study, we consider a set of coupled equations for the acoustic pressure $p$ and the acoustic velocity $\mathbf{v}=\left(v_{x}, v_{y}, v_{z}\right)$, which have been obtained by neglecting terms of $\operatorname{order}\left(\left|\mathbf{V}_{0}\right| / c\right)^{2}[10]$. These equations are given by:

$$
\begin{gathered}
\frac{\partial p}{\partial t}+\left(\mathbf{V}_{0} \cdot \nabla\right) p+\rho_{0} c^{2} \nabla \cdot \mathbf{v}=\rho_{0} c^{2} S, \\
\rho_{0} \frac{\partial \mathbf{v}}{\partial t}+\rho_{0}\left(\mathbf{V}_{0} \cdot \nabla\right) \mathbf{v}+\rho_{0}(\mathbf{v} \cdot \nabla) \mathbf{V}_{0}+\nabla_{p}=\mathbf{R},
\end{gathered}
$$


where $\mathbf{V}_{0}=\left(V_{0 x}, V_{0 y}, V_{0 z}\right)$ is the mean flow, $\rho_{0}$ is the mean density of air and $c$ is the adiabatic sound speed in the air. In the examples presented in this study, the sound speed is constant, $c=c_{0}$, where $c_{0}$ is the reference sound speed, and there is no mean flow, $\mathbf{V}_{\mathbf{0}}=\mathbf{0}$. The source terms $S$ and $\mathbf{R}$ represent respectively a mass source and external forces. The equations (1) and (2) are then written in the following conservative form:

$$
\frac{\partial \mathbf{U}}{\partial t}+\frac{\partial \mathbf{E}}{\partial x}+\frac{\partial \mathbf{F}}{\partial y}+\frac{\partial \mathbf{G}}{\partial z}+\mathbf{H}=\mathbf{B}
$$

where $\mathbf{U}=\left[p, \rho_{0} v_{x}, \rho_{0} v_{y}, \rho_{0} v_{z}\right]^{\mathrm{T}}$ is the unkown vector, $\mathbf{E}, \mathbf{F}, \mathbf{G}$ and $\mathbf{H}$ are the Eulerian fluxes and $\mathbf{B}$ is the source vector.

Non-flat boundaries are accounted for by using curvilinear coordinates (see, e.g., [15]). For that, a mapping is defined from a Cartesian mesh to a curvilinear boundary-fitted mesh, as shown in Fig. 1. The equation (3) is obtained in the curvilinear coordinate system $(\xi, \zeta, \eta)$ using chain rule expansions:

$$
\left[\begin{array}{lll}
\frac{\partial}{\partial x} & \frac{\partial}{\partial y} & \frac{\partial}{\partial z}
\end{array}\right]^{T}=\mathbf{J}\left[\frac{\partial}{\partial \xi} \frac{\partial}{\partial \zeta} \frac{\partial}{\partial \eta}\right]^{T},
$$

where $\mathbf{J}=\partial(\xi, \zeta, \eta) / \partial(x, y, z)$ is the Jacobian matrix of the transformation. This leads to:

$$
\frac{\partial \mathbf{U}^{*}}{\partial t}+\frac{\partial \mathbf{E}^{*}}{\partial \xi}+\frac{\partial \mathbf{F}^{*}}{\partial \zeta}+\frac{\partial \mathbf{G}^{*}}{\partial \eta}+\mathbf{H}^{*}=\mathbf{B}^{*}
$$
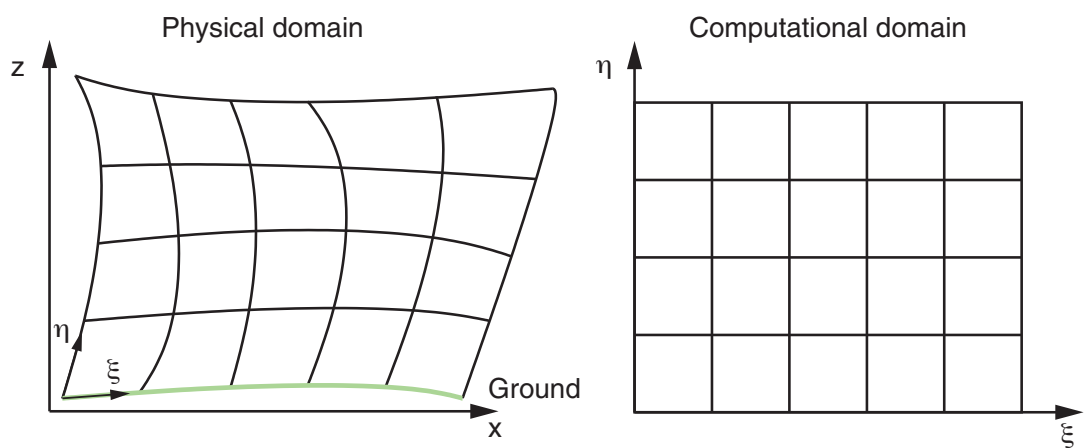

Figure 1: Coordinate transformation from the physical domain to the computational domain. From Dragna et al. [12] 
where the fluxes are $\mathbf{U}^{*}=\mathbf{U} / J, \mathbf{E}^{*}=\left(\xi_{x} \mathbf{E}+\xi_{y} \mathbf{F}+\xi_{z} \mathbf{F}\right) / J, \mathbf{F}^{*}=\left(\zeta_{x} \mathbf{E}+\zeta_{y} \mathbf{F}+\zeta_{z} \mathbf{G}\right) / J$, $\mathbf{G}^{*}=\left(\eta_{x} \mathbf{E}+\eta_{y} \mathbf{F}+\eta_{z} \mathbf{G}\right) / J, \mathbf{H}^{*}=\mathbf{H} / J$ and $\mathbf{B}^{*}=\mathbf{B} / J$. In the preceding equations, $J=|\mathbf{J}|$ is the Jacobian of the transformation. The notation $i_{j}=\partial i / \partial j$ is employed to express the partial derivatives of the coordinate functions.

\section{B. Numerical schemes}

Low-dissipation and low-dispersion schemes are required to obtain accurate numerical solutions in long range sound propagation problems. Among various possible methods to compute the spatial derivatives, finite-difference methods are probably the most common. Given an uniform mesh of spatial step $\Delta x$, the derivative of the quantity $u$ at the mesh point $x_{l}=l \Delta x$, is obtained for a centered finite-difference scheme over $2 N+1$ points by :

$$
\left.\frac{\partial u}{\partial x}\right|_{l} \simeq \frac{1}{\Delta x} \sum_{m=-N}^{N} a_{m} u_{l+m},
$$

where $a_{m}$ are the stencil coefficients with $a_{0}=0$ and $a_{-m}=a_{m}$. The stencil coefficients are deduced classically from a Taylor expansion, yielding an error decreasing as $(\Delta x)^{2 N}$. Alternative methods, based on the minimization of the errors generated by finite-difference schemes in the wavenumber space, are also widespread [16, 17]. One can cite the centered fourth-order scheme over 11 points of Bogey and Bailly [17], which has been optimized down to four points per wavelength and which is employed later.

A standard method to study the numerical errors generated by the finite difference schemes is to consider an harmonic wave $u=\exp (i k x)$, with wavenumber $k$. Eq. (6) becomes:

$$
i k u_{l} \simeq \frac{2 i}{\Delta x} \sum_{m=1}^{N} a_{m} \sin (m k \Delta x) u_{l} .
$$

The term on the right side of the preceding equation can be written as $i k^{*} u_{l}$, where $k^{*}$ is called the effective wavenumber and is given by the relation:

$$
k^{*} \Delta x=2 \sum_{m=1}^{N} a_{m} \sin (m k \Delta x)
$$

The effective wavenumber allows us to quantify easily the errors generated by the numerical schemes. For that, the simplest wave equation, which is the 1-D advection equation: 


$$
\frac{\partial u}{\partial t}+c \frac{\partial u}{\partial x}=0
$$

is considered with the initial value $u(x, t=0)=\exp (i k x)$. The solution is then given by [18]:

$$
u(x, t)=\exp \left(i k x-i k^{*} c t\right)=\exp (i k x-i k c t) \exp \left(i\left[k-k^{*}\right] c t\right) .
$$

As the effective wavenumber is real for a centered finite-difference scheme, only an error on the phase, which grows as the wave propagates, is introduced. At a mesh point $x_{l}=l \Delta x$, the signal emitted initially at $x=0$ is received with a phase error equal to $\varepsilon_{\text {phase }}=l\left[k-k^{*}\right] \Delta x$. Following Fornberg [19], one can then deduce the number of points per wavelength required to ensure a phase error lower than a chosen threshold after that the wave has propagated over a given number of wavelength. This has been displayed for a threshold of $\pi / 10$ in Fig. 2 (a), and of $\pi / 20$ in Fig. 2 (b) for various finite-difference schemes, which are the standard schemes of order 2 (denoted by FD2s), 4 (FD4s), 6 (FD6s), 8 (FD8s) and 10 (FD10s) and the optimized scheme of Bogey and Bailly [17] of order 4 over 11 points (FD4o11). It is seen that as the order of the standard scheme increases, the number of points per wavelength is reduced. Additionaly, the optimized scheme FD4o11 appears favorably compared to the standard scheme with the same number

(a)

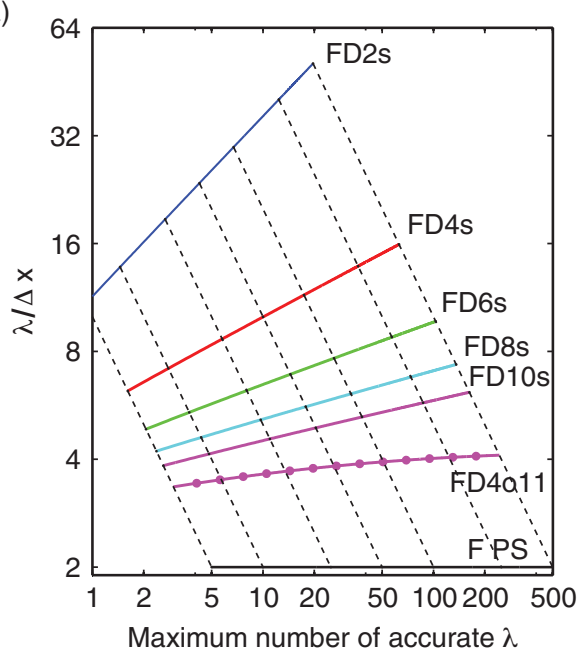

(b)

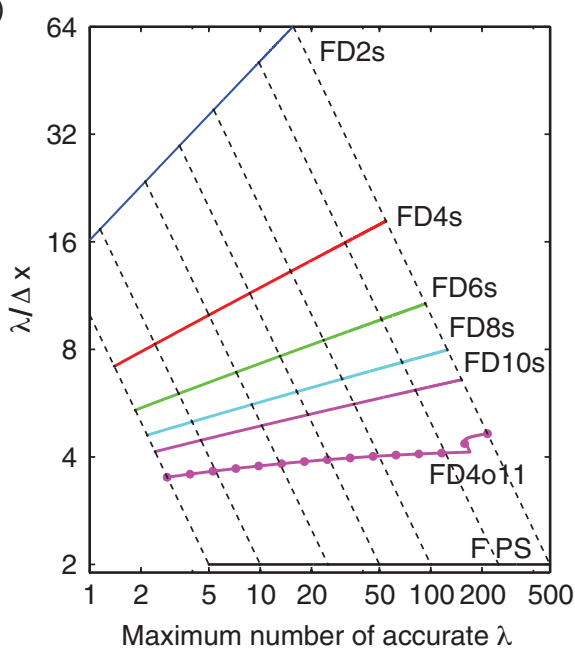

Figure 2: $\quad$ Number of points per wavelength $\lambda / \Delta x$ as a function of the maximal number of wavelength accurately resolved for a criterion (a) $\varepsilon_{\text {phase }} \leq$ $\pi / 10$ and (b) $\varepsilon_{\text {phase }} \leq \pi / 20$ for various numerical methods: FD2s (blue), FD4s (red), FD6s (green), FD8s (cyan), FD10s (magenta), FD4o11 (magenta with open circles) and Fourier PS (black). 
of stencil points, which is the standard scheme of order 10. As an exemple, propagation of an harmonic wave of wavelength $\lambda=1 \mathrm{~m}$, corresponding to a frequency $f=340 \mathrm{~Hz}$, over $100 \mathrm{~m}$ is considered. For FD2s, approximately 80 points per wavelength are required to obtain a phase error lower than $\pi / 10$, resulting at a computational domain of about 8000 points. For the other standard schemes, 17, 10, 7 and 5 points per wavelength are required for FD4s, FD6s, FD8s and FD10s, respectively. Only 4 points per wavelength are required for the optimized scheme, yielding only 400 points in the computational domain. The situation is even worse as the precision increases. Thus, to get a phase error lower than $\pi / 20$, 125 points per wavelength are now required for FD2s, while 4 points per wavelength are still sufficient for FD4o11. This demonstrate that high-order schemes are numerically efficient for long range wave propagation and that loworder shemes should be prohibited. Although not discussed here, similar considerations apply also for the time-integration scheme (see, e.g., [20, 17, 21]).

Other high-order numerical methods than finite-difference methods are also possible for spatial differentiation. For instance, pseudospectral methods based on Fourier series (see e.g. [22]) are well-spread numerical techniques for wavepropagation problems. Their major interest is that they ensure a satisfactory accuracy for waves down to two points per wavelength, which is the NyquistShannon sampling limit. They are however restricted to problems with periodic boundaries. Extensions have been proposed to account for perfectly reflecting surfaces [23] or impedance surfaces with frequency-independent properties [11]. However, frequency-dependent impedance models for naturals ground can not be accounted for up to now using the Fourier pseudospectral method, which limits its use for practical applications.

In this work, optimized finite-difference schemes and selective filters over 11 points are used to compute the spatial derivative and to remove grid-to-grid oscillations, respectively. For the interior points, the centered fourth-order finite-difference scheme of Bogey and Bailly [17] and the sixth-order selective filter of Bogey et al. [24] are chosen. For the boundary points, the non-centered finite-difference schemes and the non-centered selective filters of Berland et al. [18] are used. The filtering coefficient is set to 0.2 for all filters except at the end points at which the filtering coefficient is 0.01 . The time integration is performed with an optimized six-step fourth-order Runge-Kutta algorithm [21].

\section{Time-domain impedance boundary condition}

For sound propagation over natural ground, the local reaction approximation is generally applicable (see e.g. [4]). Under this assumption, the impedance boundary condition is written in the frequency domain as $P(\omega)+Z(\omega) V_{n}(\omega)=0$, where $\omega$ is the angular frequency, $P$ and $V_{n}$ are the Fourier transforms of the pressure $p$ and of the acoustic velocity normal to the ground $v_{n}$. For a rigid ground, $v_{n}=0$ and the surface impedance is infinite. The translation of the impedance boundary condition into the time domain leads to the convolution: 


$$
p(t)=-\int_{-\infty}^{t} z\left(t-t^{\prime}\right) v_{n}\left(t^{\prime}\right) \mathrm{d} t^{\prime}
$$

where $z(t)$ is the inverse Fourier transform of the surface impedance. Not all surface impedance models are physically possible [25]. Indeed, $\not(t)$ must be real and causal. Moreover, because the ground absorbs energy, one must have $\operatorname{Re}[Z(\omega)]>0$, for $\omega>0$, where Re denotes the real part. In [26], these conditions have been checked for various impedance models widely used in the outdoor sound propagation community. It has been shown, for instance, that the Delany and Bazley impedance model [27], which is probably the most widespread model in outdoor sound propagation studies, can not be causal and real simultaneously and is thus not suitable for time-domain computations.

The computation of convolution in Eq. (11) is not straightforward. Indeed, a direct evaluation of the convolution would require to store the acoustic velocity at the ground at each iteration. This would be inefficient for long range sound propagation in a 3-D environment. Therefore, a time-domain boundary condition (TDBC) $[28,29,30]$, based on a recursive convolution approach, is used to account for an impedance ground surface. The TDBC requires the approximation of the impedance in the frequencydomain by a rational function:

$$
Z(\omega) \approx Z_{\infty}+\sum_{k=1}^{N} \frac{A_{k}}{\lambda_{k}-i \omega},
$$

where $Z_{\infty}$ is the limit value of $Z(\omega)$ as $\omega$ tends to infinite, $\lambda_{k}$ are the poles, $N$ is the number of poles and $A_{k}$ are numerical coefficients. The parameters $\lambda_{k}$ and $A_{k}$ can be obtained by using different methods [30], such as the vector fitting algorithm [31]. The rational function is passive and causal if the poles $\lambda_{k}$ are real or complex conjugates and have positive real parts [29]. The condition $\operatorname{Re}[Z(\omega)]>0$ for $\omega>0$ must be checked for each set of coefficients $\left(A_{k}, \lambda_{k}\right)$.

For simplicity, only real poles are considered hereafter. A detailed presentation of the TDBC can be found in [29]. Assuming that the normal acoustic velocity is constant over a time step $\Delta t$, the values of the acoutic pressure and velocity at the ground at the discretized time $m \Delta t$, respectively denoted by $p^{(m)}$ and $u_{n}^{(m)}$, are related by the formula.

$$
p^{(m)}=-Z_{\infty} v_{n}^{(m)}+\sum_{k=1}^{N} A_{k} \phi_{k}^{(m)},
$$

where $\phi_{k}^{(m)}$ are called the accumulators. They are computed using the recursive expression:

$$
\phi_{k}^{(m)}=-v_{n}^{(m)} \frac{1-e^{-\lambda_{k} \Delta t}}{\lambda_{k}}+\phi_{k}^{(m-1)} e^{-\lambda_{k} \Delta t} .
$$



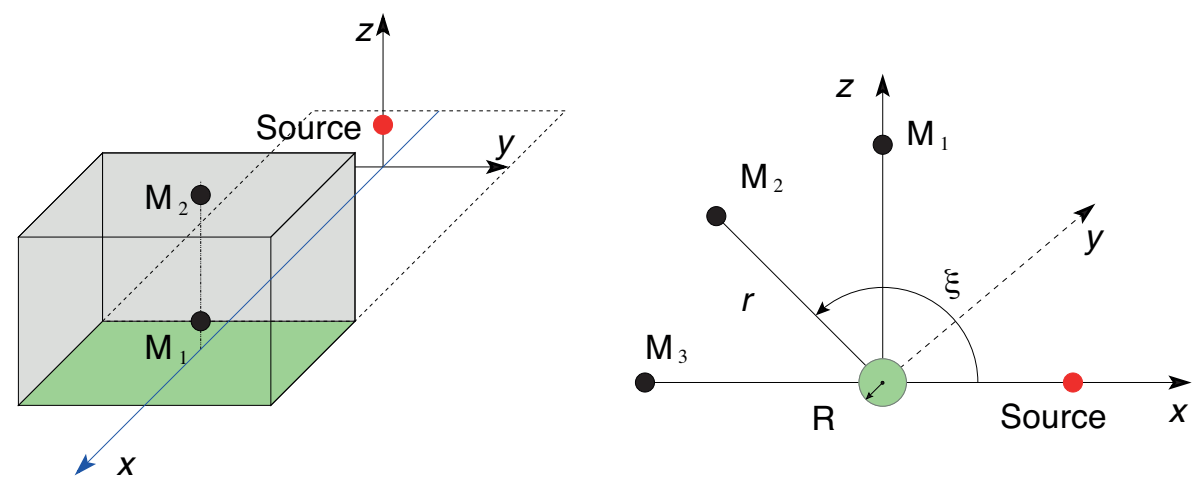

Figure 3: Schematic of the two test cases: (a) long-range propagation over an impedance plane and (b) diffraction from an impedance sphere. Taken in part from reference [12]

Thus, the recursive convolution method requires only two storage locations per accumulator, independently of the number of time steps, while a direct computation of the convolution would demand a much larger memory space. This is, therefore, an efficient method for long range propagation over impedance surfaces.

\section{VALIDATION TEST CASES}

Two test cases dealing with the diffraction of acoustic waves are performed to demonstrate the ability of taking into account impedance surfaces. The first one is concerned with long range propagation over a flat ground. The second one corresponds to diffraction of acoustic waves by an impedance sphere. The schematic of these problems is displayed in Figs. 3.

In both cases, three different boundary conditions are considered. The first one is a perfectly reflecting surface, corresponding to an infinite surface impedance. The other ones are impedance surfaces, using the one-parameter Miki impedance model [32]. The second surface impedance aims at modeling a semi-infinite ground layer with an air flow resistivity $\sigma_{0}=100 \mathrm{kPa}$.s.m ${ }^{-2}$, which represents a grassy ground, and the third one a rigidly backed layer of thickness $d=10 \mathrm{~cm}$ and of air flow resistivity $\sigma_{0}=10 \mathrm{kPa} . \mathrm{s} . \mathrm{m}^{-2}$, which represents a snowy ground. The source is a Gaussian impulse, obtained by initializing the simulation with $\mathbf{v}(\mathbf{x}, t=0)=\mathbf{0}$ and $p(\mathbf{x}, t=0)=\rho_{0} c_{0}^{2} Q\left(\mathbf{x}-\mathbf{x}_{\mathrm{S}}\right)$, where $\mathbf{x}_{\mathrm{S}}$ is the position of the center of the source and

$$
Q(\mathrm{x})=\exp \left(-\ln (2) \frac{x^{2}}{B_{x}^{2}}\right)
$$

with $x=|\mathbf{x}|$. 
A. Long range sound propagation in an homogeneous atmosphere In this first test case, long range propagation above a flat ground is studied. The center of the source is located initially at $\mathbf{x}_{\mathbf{S}}=\left(0,0, z_{S}\right)$, with $z_{S}=1 \mathrm{~m}$. As an impulse source is considered, the spatial extent of the acoustic signal is small. Therefore, the computational domain can be reduced to a narrow region near the impulse signal. As proposed by Salomons et al. [33], a moving frame which follows the propagation of the impulse signal is implemented. The mesh is uniform and the spatial step is set to $\Delta x=\Delta y=\Delta z=0.05$ $\mathrm{m}$. The CFL number, defined by CFL $=c_{0} \Delta t / \Delta x$, is 0.67 and 9000 time iterations are performed. Perfectly matched layers [34] are used at the outter boundaries. The computational domain has $345 \times 501 \times 396$ points and its size is $[-15 \mathrm{~m}, 2.25 \mathrm{~m}] \times[-12.5$ $\mathrm{m}, 12.5 \mathrm{~m}] \times[0 \mathrm{~m}, 19.75 \mathrm{~m}]$ in the $x-, y$-and $z$-directions, respectively.

The waveforms obtained from the numerical solution and from an analytical solution $[35,12]$ are displayed in Fig. 4 as a function of the normalized time $\bar{t}=c_{0} t / x$ for the different boundary conditions and for two receivers $\mathrm{M}_{1}(x=200 \mathrm{~m}, y=0 \mathrm{~m}, z=2 \mathrm{~m})$ and $\mathrm{M}_{2}(x=200 \mathrm{~m}, y=0 \mathrm{~m}, z=10 \mathrm{~m})$. For the perfectly reflecting surface, the waveforms are quite simple. As the receivers are in grazing incidence, the direct and reflected waves are mixed. Therefore, only one contribution can be seen. Note that the time of arrival of the impulse signal at the receiver $\mathrm{M}_{2}$ is slightly larger than that at the receiver $\mathrm{M}_{1}$, because of the larger travel time. For the impedance surfaces close to the ground, the waveforms are dominated by a low-frequency oscillating wave. This is in particular the case for the snowy ground, for which the spatial extent of this wave exceeds the length of the computational domain. Therefore, it is seen that the numerical solution at $\mathrm{M}_{1}$ abruptly goes to 0 for
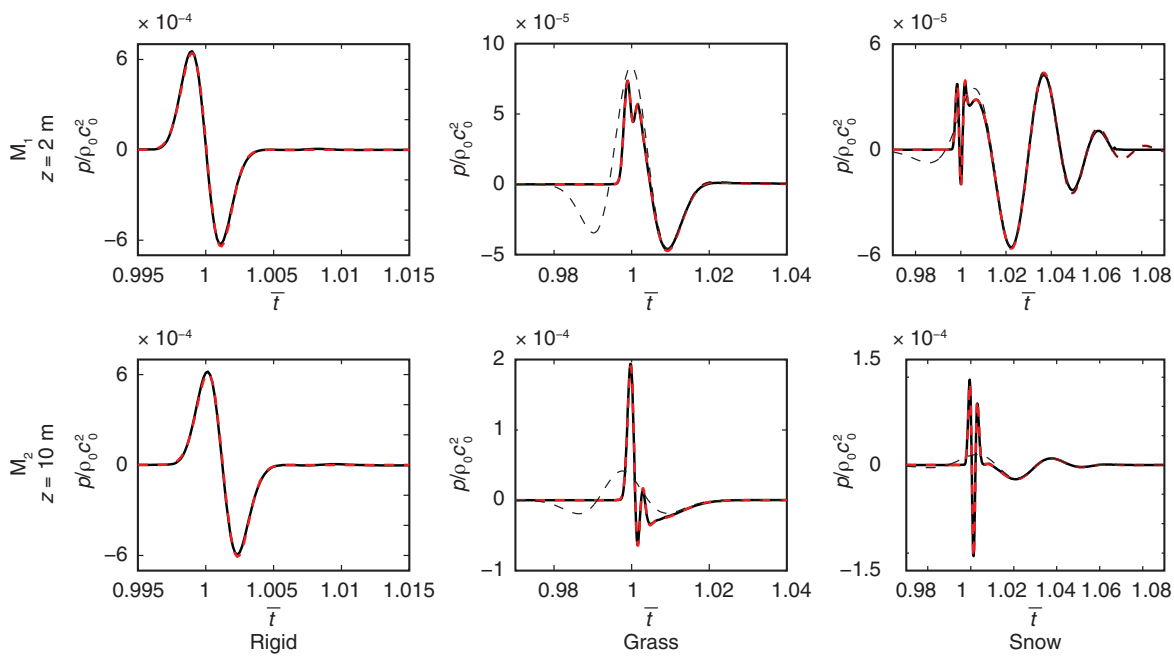

Figure 4: Waveforms of the normalized pressure $p / \rho_{0} c_{0}^{2}$ obtained at receivers $\mathbf{M}_{1}$ and $\mathrm{M}_{2}$ and for the three boundary conditions as a function of the normalized time $\bar{t}=c_{0} t / x$ : numerical (black solid line) and analytical (red dashed line) solutions and (black dashed line) surface wave solution. 
$\bar{t}=1.07$. This behaviour is expected to be related to the surface wave component. To confirm this assumption, the surface wave solution has been plotted in a black dashed line in Fig. 4. It matches the oscillating part of the waveform with a good agreement. For the receiver $M_{2}$ located at a higher altitude than $M_{1}$, the contribution of the surface wave is reduced, as its amplitude decays exponentialy with the height above the ground. These surface waves have been also exhibited in results of two-dimensional simulations of long range propagation over impedance surfaces in [36]. Note that in all cases, the analytical and numerical solutions are superimposed.

\section{B. Diffraction by an impedance sphere}

The second test-case deals with diffraction of spherical waves by an impedance sphere. It aims to demonstrate that the TDBC is also efficient for non-flat geometries. The coordinate transformation

$$
\begin{gathered}
x=(1+\eta) \cos \xi, \\
y=(1+\eta) \sin \zeta \sin \xi, \\
z=(1+\eta) \cos \zeta \sin \xi,
\end{gathered}
$$

is used. In this section, all spatial parameters are made non-dimensional using the sphere radius $R$ as the length scale. Note that $(r=1+\eta, \xi, \zeta)$ correspond to the spherical coordinates, with $x$-axis as the polar axis. The Gaussian pulse is centered at $\mathrm{x}_{S}=\left(x_{S}, 0,0\right)$ with $x_{S}=3$. The Gaussian halfwidth is $B_{x}=0.4$. Three receivers denoted as $\mathbf{M}_{1}, \mathbf{M}_{2}$ and $\mathbf{M}_{3}$ are placed in the computational domain with $r=10, \zeta=0$ and with respectively $\xi=\pi / 2, \xi=3 \pi / 4$ and $\xi=\pi$. Periodic boundary conditions are imposed in the polar and azimuthal directions, i.e. in the $\xi$ and $\zeta$-directions. In the radial direction, i.e. in the $\eta$-direction, the non-reflecting boundary condition of Bogey and Bailly [37] is used.

The Jacobian of the transformation:

$$
J=\frac{1}{(1+\eta) \sin \xi},
$$

is singular at $\xi=0$ and at $\xi=\pi$. To avoid these singularities, as proposed by [38] for the polar case, the mesh is shifted by $\Delta \xi / 2$ in the $\xi$-direction. Additionaly, the mesh in the azimuthal direction is very coarse close to $\xi=0$ and at $\xi=\pi$, which would result to a very small time step due to the CFL stability restriction and hence to a large computational cost. Therefore, following Bogey et al. [39], the discretization in the $\zeta$-direction is artificially increased by evaluating the derivative not from directly adjacent points but from points separated by $m \Delta \zeta$ avec $m>1$. Details on the numerical methods used for this test case can be found in Dragna et al. [12]. $160 \times 320 \times 600$ points are used respectively in the $\xi, \zeta$ and $\eta$ directions with mesh sizes $\Delta \xi=\Delta \zeta=$ 0.02 and $\Delta \eta=0.025$. The CFL number is set to 0.7 , and 4500 time iterations are performed. 

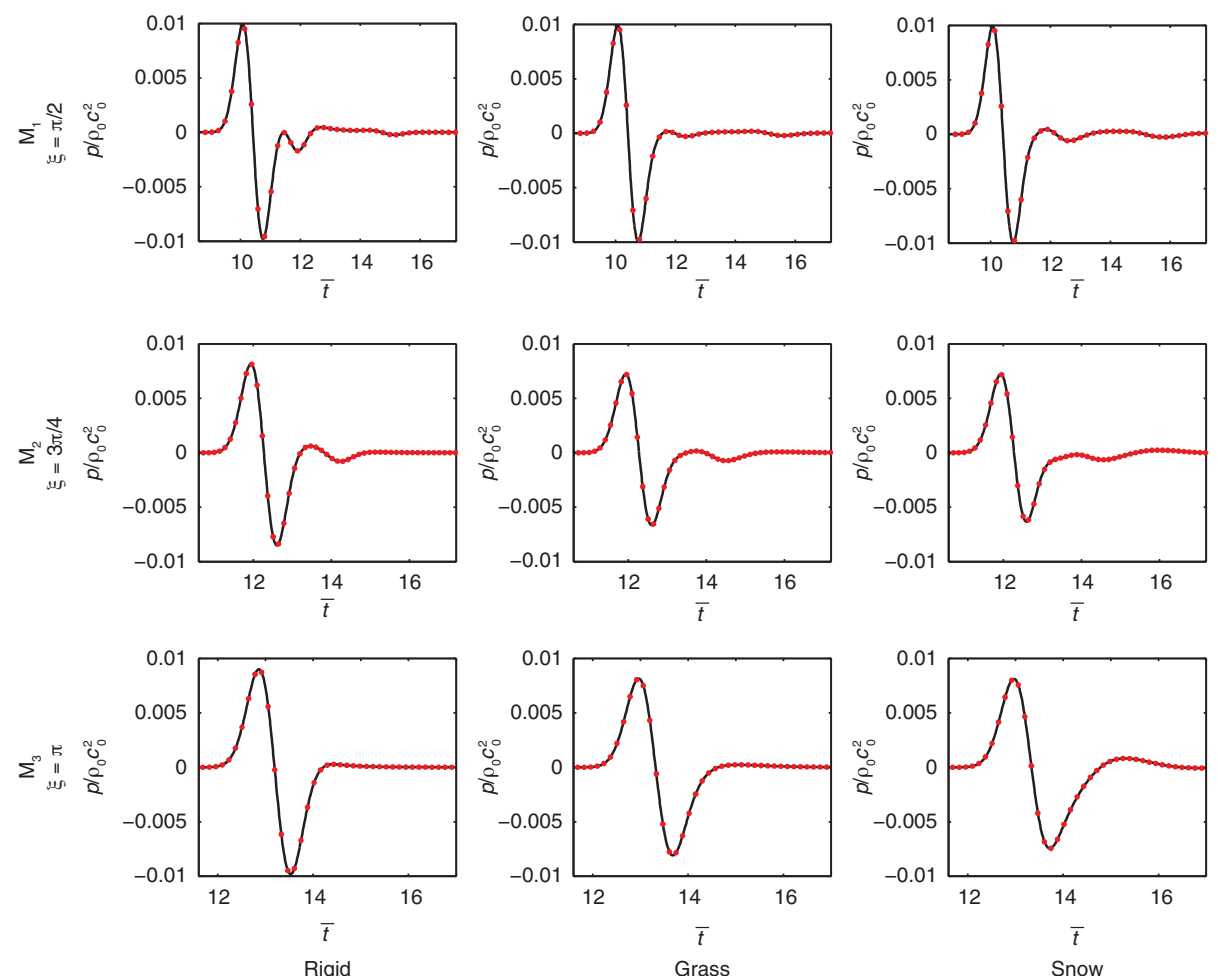

Figure 5: Waveforms of the normalized pressure $p / \rho_{0} c_{0}^{2}$ obtained for the receivers $\mathrm{M}_{1}, \mathrm{M}_{2}$ and $\mathrm{M}_{3}$ and for the three boundary conditions as a function of the normalized time $\bar{t}=c_{0} t / R$ : Numerical (black solid line) and analytical (red dots) solutions. From Dragna et al. [12].

In Fig. 5, the pressure waveforms obtained at the three receivers for the three boundary conditions are displayed as a function of the time. It is seen that the analytical solution is perfectly superimposed on the numerical solution in all cases. Therefore, it is demonstrated that the TDBC is well-adapted to account for impedance surfaces in a non-flat geometry in the time domain.

\section{APPLICATION TO BROADBAND MOVING SOURCES}

The numerical solver presented in the previous section is now used to investigate the radiation by moving sources in a realistic environment.

\section{A. Implementation}

The implementation of a moving source in motion in discretization methods is not straightforward. As an example, the simplest acoustic sources which are point-sources, 
are difficult to handle when they are moving. Indeed, at each time step, they must be located on a grid node, which requires special attention for the grid generation. Moreover, it raises an another issue for explicit time-integration schemes. Indeed, let us consider a point-source in motion at an uniform speed $V_{0}$. During one time step $\Delta t$, the source must move at least by one spatial step $\Delta x$. In addition, the time step must satisfy the Courant-Friedrichs-Lewy condition to ensure the numerical stability. This writes $\mathrm{CFL}<\mathrm{CFL}_{\max }$, where $\mathrm{CFL}_{\max }$ is the CFL limit. The Mach number of the source, defined by $M=V_{0} / c_{0}$, is then related to the CFL number by the relation $M=1 / \mathrm{CFL}$, which shows that the source speed can not be chosen too small. This dramatically limits possible applications in transportation noise. A way to overcome these issues is to consider an extended acoustic source. If the source is compact, it is expected that it behaves as a point-source. Details on the effects of an extended source in motion at an uniform speed on the acoustic field can be found in [40].

Implementation of a monopole-type moving source is performed trough the mass source term in Eq. (1) by setting :

$$
S(\mathbf{x}, t)=Q\left(\mathbf{x}-\mathbf{x}_{\mathbf{S}}(t)\right) s(t),
$$

where $Q$ is the source spatial distribution and $\mathbf{x}_{\mathbf{S}}(t)$ is the position of the source center at time $t$. For dipole-type sources, it is preferable to use the external forces term in Eq. (2). The time-domain signal $s(t)$ is obtained by multiplying the Fourier transform of a synthetized white noise signal by the desired spectrum in the frequency domain and by doing an inverse Fourier transform of the result. For each realization of the source signal, one computation has to be performed. The mean value of an acoustic quantity is thus obtained by computing its average value over the number of realizations of the source signal. Increasing the number of realizations improves the convergence of the results but leads to an additional computational cost. A trade-off has then to be found to ensure a satisfactory convergence and an acceptable computational cost.

In following cases, sources are moving at a constant height and at a constant speed and, hence, $\mathbf{x}_{\mathbf{S}}(t)=\left(V_{0} t, 0, z_{S}\right)$. The source spatial distribution is Gaussian, with $B_{x}=0.1 \mathrm{~m}$ (see Eq. (15)). In addition, the one-sided power spectral density (PSD) of $s(t)$ is a Gaussian function:

$$
S_{s s}=s_{0} \exp \left\lceil-2 \frac{\left(f-f_{c}\right)^{2}}{f_{b}^{2}}\right\rceil,
$$

where $s_{0}$ is a normalization parameter set to 1.07 . The central frequency $f_{c}$ is $300 \mathrm{~Hz}$. The parameter $f_{b}$ controls the decrease of the Gaussian and is set to $100 \mathrm{~Hz}$. The frequency content of the source is thus significant for frequencies between $200 \mathrm{~Hz}$ and $400 \mathrm{~Hz}$. As for the maximum frequency of interest $f=400 \mathrm{~Hz}$, the parameter $k_{0} B$ is less than 1 , the source can be considered as compact and is expected to behave like a point source. Ten realizations of the random source signal are performed for each case. 


\section{B. Validation}

First, radiation by a source moving at a constant above a perfectly reflecting ground is considered as a test case to validate the proposed methodology. A schematic of the problem is depicted in Figure 6. The source is moving along the $x$-axis at a constant height $z_{S}=2.1 \mathrm{~m}$ and at a constant speed $V_{0}=50 \mathrm{~m} \cdot \mathrm{s}^{-1}$. The Mach number is then equal to 0.15 . At the initial simulation time, the source is located at $x=-95 \mathrm{~m}$. The grid has $2001 \times 351 \times 72$ points. The mesh grid is uniform and $\Delta x=\Delta y=\Delta z=0.1 \mathrm{~m}$. The domain size is then $[-100 \mathrm{~m} ; 100 \mathrm{~m}] \times[-5 \mathrm{~m} ; 30 \mathrm{~m}] \times[0 \mathrm{~m} ; 7.1 \mathrm{~m}]$. The CFL number is set to unity and 12000 time iterations are performed. Two receivers denoted as $\mathrm{M}_{1}$ and $\mathrm{M}_{2}$ and located respectively at $x=0 \mathrm{~m}, y=4.9 \mathrm{~m}$ and $z=3 \mathrm{~m}$ and at $x=$ $0 \mathrm{~m}, y=24.9 \mathrm{~m}$ and $z=3.5 \mathrm{~m}$ are considered. The instantaneous PSD obtained at these receivers are plotted as a function of the time and the frequency in Figure 7 . The reference pressure is set to $p_{\text {ref }}=2 \times 10^{-5} \mathrm{~Pa}$. The Doppler shift is clearly observed, as the acoustic pressure has a higher frequency content when the source approaches the receiver than when the source recedes from the receiver. As for a non-moving source, the ground effects are important. As an illustration, strong destructive and constructive interferences are clearly visible.

In the case of reflection by a perfectly reflecting surface, the acoustic field is the sum of two contributions, the first one due to the source and the second one due to an image source located symmetrically to the source with respect to the ground plane. As the analytical solution of the acoustic pressure for a point source moving at a constant speed in free field is well-known [41], the analytical solution for a harmonic point source moving at a constant speed above a perfectly reflecting surface and emitting at the angular trequency $\omega_{0}$ is thus easily obtained, yielding:

$$
\begin{aligned}
& p_{0}(\mathrm{x}, t)=\rho_{0} i \omega_{0} \exp \left(-i \omega_{0} t\right) \\
& {\left[\frac{\exp \left(i k R_{\mathrm{e}, 1}\right)}{4 \pi R_{\mathrm{e}, 1}\left(1-M_{0} \cos \theta_{\mathrm{e}, 1}\right)^{2}}+\frac{\exp \left(i k R_{\mathrm{e}, 2}\right)}{4 \pi R_{\mathrm{e}, 2}\left(1-M_{0} \cos \theta_{\mathrm{e}, 2}\right)^{2}}\right] .}
\end{aligned}
$$

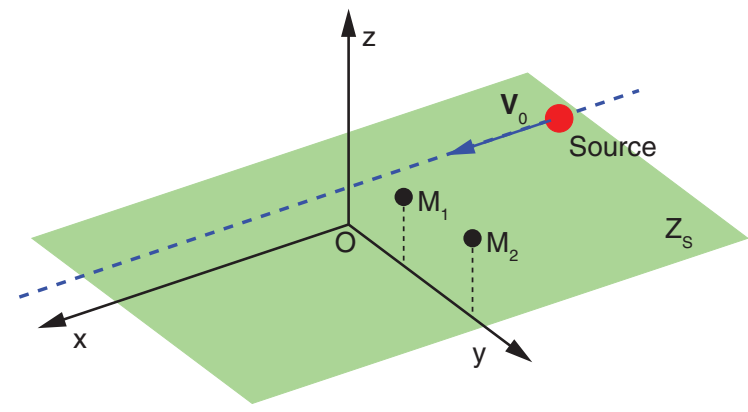

Figure 6: $\quad$ Source moving above a flat surface of impedance $Z_{S}$ along the $x$-axis at a constant speed $\mathbf{V}_{\mathbf{0}}$ in a 3-D geometry. Two receivers $\mathrm{M}_{1}$ and $\mathrm{M}_{2}$ are considered. From Dragna et al. [40]. 
(a)

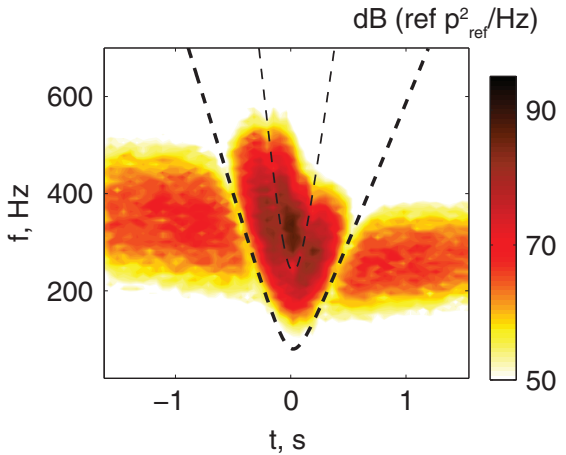

(b)

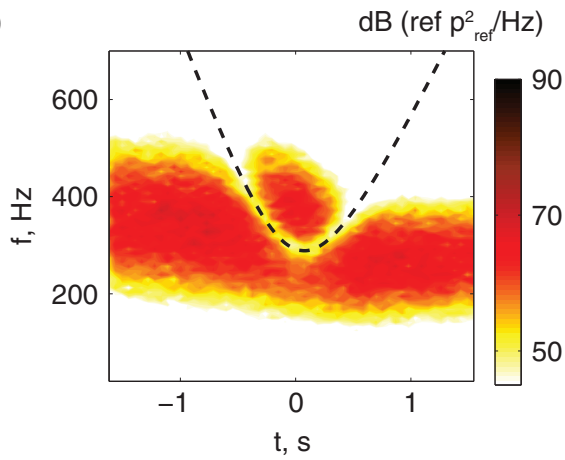

Figure 7: Instantaneous PSD of the acoustic pressure in $\mathrm{dB}\left(\operatorname{ref} p_{\mathrm{ref}}^{2} / \mathrm{Hz}\right)$ as a function ofthe time and the frequency obtained from the numerical solution at the receiver (a) $M_{1}$ and (b) $M_{2}$. The dashed lines represent the locations of destructive interference. From Dragna et al. [40].

In the preceding equation, $\left(R_{e, 1}, \cos \theta_{e, 1}\right)$ and $\left(R_{e, 2}, \cos \theta_{e, 2}\right)$ are the retarded time coordinates whose origin is the source and the image source, respectively. Minima of the acoustic pressure modulus occur when $k_{n}\left(R_{e, 2}-R_{e, 1}\right)=(1+2 n) \pi$, for $n$ positive integer. The destructive interference is directly linked to the frequency $f_{n}=c_{0} k_{n} /(2 \pi)$. However, due to the Doppler shift, it does not appear from the observer's point of view at the frequency $f_{n}$. Moreover, the signal originated from the source and the image source are not perceived at the same frequencies for a fixed observer, because their locations are not the same. However, as the source is close to the ground, and, hence, $\cos \theta_{e, 2} \approx \cos \theta_{e, 1}$, the frequency at the observer is approximately equal to $f_{D, n}=f_{n} /$ ( 1 $\left.-M_{0} \cos \theta_{e, 1}\right)$. The curves representing $f_{D, n}$ for $n=0$ at the two receivers and for $n=$ 1 at the receiver $M_{1}$ are plotted in a dashed line in Figure 7. A good agreement is found for the destructive interference location. Other values of $n$ are related to higher frequencies which are not relevant for the source considered here.

The numerical solution is now compared to an analytical solution. From Eq. (22), the instantaneous PSD for a broadband spherical source with a spherical symmetry moving above a rigid ground is given by:

$$
\begin{aligned}
S_{p p}(\mathbf{x}, f, t) & =S_{s s}(f) \rho_{0}^{2} \omega^{2}\left|\hat{Q}\left(k_{D}\right)\right| \\
& \left|\frac{\exp \left(i k R_{\mathrm{e}, 1}\right)}{4 \pi R_{\mathrm{e}, 1}\left(1-M_{0} \cos \theta_{\mathrm{e}, 1}\right)^{2}}+\frac{\exp \left(i k R_{\mathrm{e}, 2}\right)}{4 \pi R_{\mathrm{e}, 2}\left(1-M_{0} \cos \theta_{\mathrm{e}, 2}\right)^{2}}\right|^{2} .
\end{aligned}
$$

In the preceding equation, $\hat{Q}$ is the spatial Fourier transform of the source spatial distribution $Q$ evaluated at wavenumber $k_{D}=k_{0} /\left(1-M_{0} \cos \theta_{e, 1}\right)$. It accounts for the 


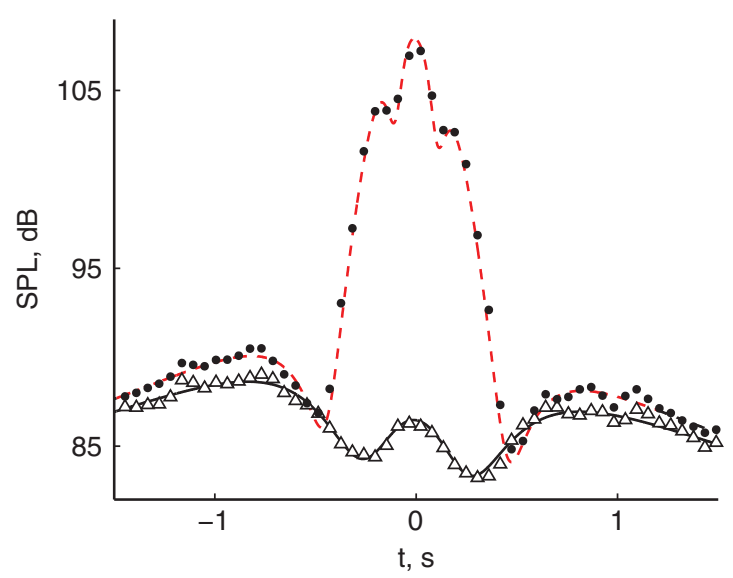

Figure 8: Instantaneous $\mathrm{SPL}$ in $\mathrm{dB}$ as a function of the time obtained from the analyticalsolution at $\mathrm{M}_{1}$ (red dashed line) and $\mathrm{M}_{2}$ (black solid line) and from the numerical solution at $\mathrm{M}_{1}$ (dots) and $\mathrm{M}_{2}$ (triangles). From Dragna et al. [40].

effect of the source spatial distribution on the acoustic field. The instantaneous sound pressure level (SPL), which is obtained by integrating the one-sided power spectral densities over frequencies:

$$
\operatorname{SPL}(\mathbf{x}, t)=\int_{0}^{+\infty} S_{p p}(\mathbf{x}, f, t) \mathrm{d} f
$$

is displayed in Figure 8 as a function of the time for the analytical and numerical solutions. It is seen that the time variations of the SPL are large at the receiver $\mathrm{M}_{1}$. Indeed, due to the constructive interference pattern, there is almost $20 \mathrm{~dB}$ difference in the SPL when the source is in front of the receiver and when the source is far from the receiver. For the receiver $\mathrm{M}_{2}$, the amplitude of the variations of the SPL is reduced. The SPL computed from the analytical and numerical solutions are in a very good accordance, as the maximum difference is about $0.5 \mathrm{~dB}$.

\section{Application on a realistic site}

The methodology presented in the preceding section is now applied to investigate the acoustic radiation by moving sources in a realistic environment. The site corresponds to a railway site with a ballasted track in La Veuve, near Reims in France, where outdoor acoustic measurements were performed in May 2010. Comparisons between experimental results and numerical predictions are presented in Dragna et al. [42]. The topography of the site is invariant in the $x$-axis. The topographic cross-section is shown in Fig. 9. The terrain is non-flat and presents, in particular, a gap for $y=18 \mathrm{~m}$, whose depth is approximately $0.8 \mathrm{~m}$. Moreover, the ground is inhomogeneous, as three different ground types corresponding to a ballast bed, a soil and a grassy ground, were 


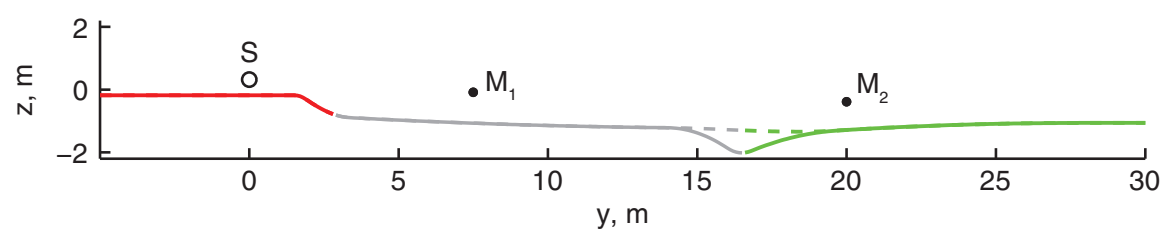

Figure 9: Topography of the site implemented in the solver with (full line) and without (dashed line) the gap. Each color corresponds to a different type of ground: ballast (red), soil (gray) and grass (green).

distinguished. The ballast bed is modeled using the Hamet and Bérangier impedance model [43] for a semi-infinite layer of air flow resistivity $0.3 \mathrm{kPa} . \mathrm{s} . \mathrm{m}^{-2}$, tortuosity 1.12 and porosity 0.5 . The soil and the grassy ground are modeled using the one-parameter Miki impedance model [32] of a rigidly backed layer of air flow resistivity $600 \mathrm{kPa} . \mathrm{s} . \mathrm{m}^{-2}$ and thickness $0.006 \mathrm{~m}$ and of air flow resistivity $180 \mathrm{kPa} . \mathrm{s} . \mathrm{m}^{-2}$ and thickness $0.018 \mathrm{~m}$, respectively.

The grid is similar to that of the previous simulation. The CFL number is set to 0.4 and 16000 time iterations are performed. The source is moving along the $x$-axis at a speed $V_{0}=100 \mathrm{~m} \cdot \mathrm{s}^{-1}$ and at a height $z_{S}=0.32 \mathrm{~m}$, corresponding to $0.5 \mathrm{~m}$ above the ballast surface. The coordinate transformation is:

$$
\begin{gathered}
x=\xi, \\
y=\zeta, \\
z=\eta+\mathrm{H}(\zeta),
\end{gathered}
$$

where $H$ is the ground profile. In order to investigate the effects of the gap, two simulations in which the gap is accounted for or not are performed. Corresponding ground profiles are displayed in Fig. 9.

Firstly, the results obtained for the ground profile with the gap are considered. The instantaneous Power Spectral Density (PSD) of the acoustic pressure at two receivers located at $\mathrm{M}_{1}(x=0 \mathrm{~m}, y=7.5 \mathrm{~m}, z=0.13 \mathrm{~m})$ and $\mathrm{M}_{2}(x=0 \mathrm{~m}, y=20 \mathrm{~m}, z=-$ $0.39 \mathrm{~m}$ ) are displayed as a function of the frequency and the time in Fig. 10. The PSD at $\mathrm{M}_{1}$ is typical of a moving source. The frequency content of the acoustic pressure is centered around $f=400 \mathrm{~Hz}$ as the source approaches the receiver ( $t<0 \mathrm{~s})$ and around $f=250 \mathrm{~Hz}$ as the source recedes from the receiver $(t>0 \mathrm{~s})$. The Doppler effect is more pronounced than in Figs. 7 (a) and (b) as the source speed is greater and as there is no noticeable interference patterns in this case. The PSD at the receiver $\mathrm{M}_{2}$, located just after the gap, is similar to the previous one. The Doppler shift occurs over a larger time, as the receiver $M_{2}$ is located at a larger distance from the source than $M_{1}$. It is seen that the PSD is lower over all the frequency band of interest as the source approaches the receiver than as the sources recedes from the receiver. 
(a)

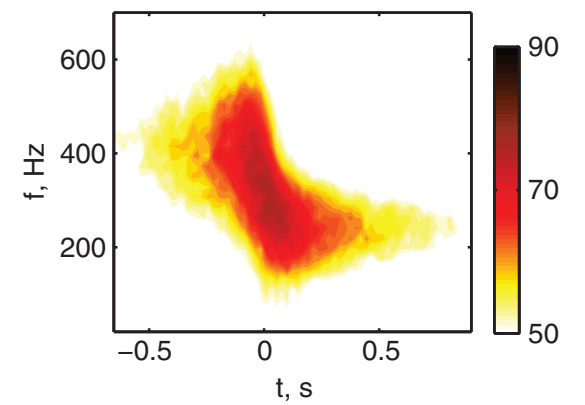

(b)

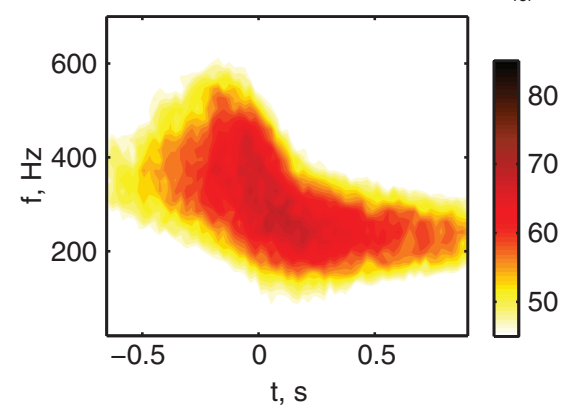

Figure 10: Instantaneous PSD of the acoustic pressure in $\mathrm{dB}\left(\right.$ ref $\left.p_{\mathrm{ref}}^{2} / \mathrm{Hz}\right)$ as a function of the time and the frequency at the receiver $M_{1}$ (a) and $M_{2}$ (b), obtained for the topography with the gap.

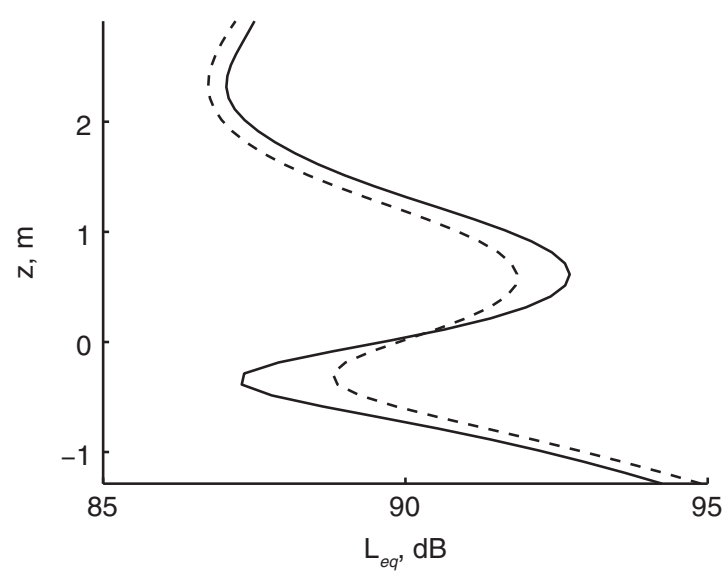

Figure 11: Equivalent sound pressure level integrated over $1 \mathrm{~s}$ obtained for the topography with (full line) and without (dashed line) the gap as a function of $z$ for a column of receivers located at $x=0 \mathrm{~m}$ and $y=20 \mathrm{~m}$.

The effects of the gap are now investigated by examining the acoustic field at a column of receivers located just after the gap, at $x=0 \mathrm{~m}$ and $y=20 \mathrm{~m}$. The equivalent sound pressure level:

$$
L_{\text {eq }}(\mathbf{x})=\frac{1}{2 t_{p}} \int_{-t_{p}}^{t_{p}} p^{2}(\mathbf{x}, t) \mathrm{d} t
$$

with $t_{p}=0.5 \mathrm{~s}$, is displayed as a function of $z$ in Fig. 11. For receivers with $z<0 \mathrm{~m}$, the sound pressure level is reduced due to the presence of the gap. As the opposite, for higher 


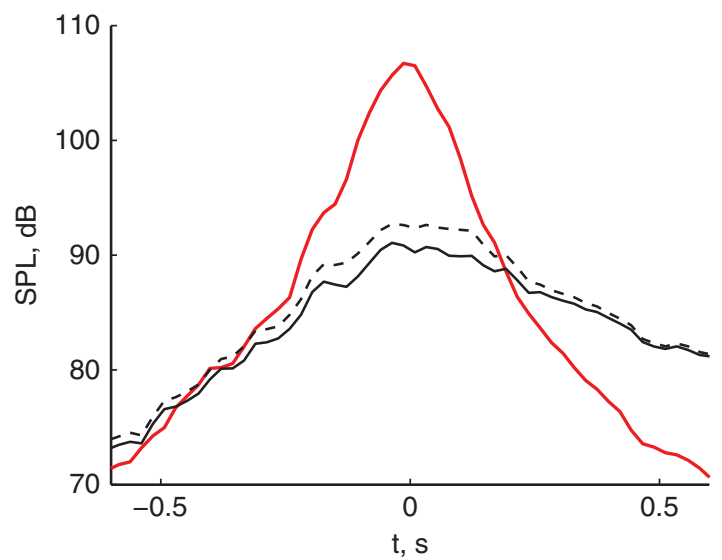

Figure 12: Instantaneous SPL in $\mathrm{dB}$ as a function of the time at $\mathrm{M}_{1}$ (red) and $\mathrm{M}_{2}$ (black) for the topography with (full line) and without (dashed line) the gap.

heights, it is increased. The maximal deviation is obtained at the receiver $\mathrm{M}_{2}$ at $z=-0.39$ $\mathrm{m}$ and is equal to $1.5 \mathrm{~dB}$.

At last, the SPL obtained at the two receivers are represented as a function of the time in Fig. 12. The receiver $M_{1}$, is located between the source and the gap. Therefore, the curves obtained for both ground profiles are superimposed and the gap has no noticeable effect. The SPL is maximum when the source is in front of the receiver, and decreases as the distance to the receiver increases. At $\mathrm{M}_{2}$, the curves representing the SPL are flatter. They are also non-symmetric around $t=0 \mathrm{~s}$, as observed previously for the time-frequency decompositions in Fig. 10 (b). This is due to the source motion and, in particular, to the convective amplification and also to the ground attenuation which depends on the frequency content of the incident waves. As the frequencies of the acoustic waves impacting the ground are not the same when the sources approaches or recedes from the receiver, the ground effect is dramatically modified. The effects of the gap are pronounced when the source is in front of the receiver. Thus, there are more than $2 \mathrm{~dB}$ difference for $t=0 \mathrm{~s}$. When the source is far from the receiver, the effects of the gap are reduced and for $|t|>0.3 \mathrm{~s}$, the curves obtained for the cases with and without the gap are almost superimposed. This behavior can be explained as diffraction effects are not the same depending on the source position relative to the receiver position. Indeed, when the source is in front of the receiver, the length of the gap along the sound propagation path is approximately $3 \mathrm{~m}$, as seen in Fig. 9. As the distance from the receiver increases, the length of the gap along the sound propagation path increases. It can then be expected that diffraction effects are significant when the source is in front of the receiver, corresponding to $t=0 \mathrm{~s}$, and decrease as the distance from the receiver increases. 


\section{CONCLUSION}

A numerical solver of the linearized Euler equations has been developed with the aim of accounting for acoustic radiation by moving sources in outdoor environments. It uses highorder finite-difference schemes for the spatial derivation and a high-order Runge-Kutta algorithm for the time-integration. An impedance boundary condition based on a recursive convolution approach is implemented to account for reflexion of acoustic waves on ground surfaces. The solver has been first validated against test cases dealing with long range propagation above an impedance plane and diffraction by an impedance sphere. It has been then employed to study radiation by moving sources. In the simple configuration of a broadband monopole-type source in motion at a constant height and at a constant speed above a perfecly reflecting ground, the time-frequency decompositions have shown strong destructive interference patterns as for a fixed source. Deviations from an analytical solution were shown to be small. The more complex configuration of a broadband source moving at a constant height and at a constant speed above a non-flat terrain, presenting a gap and with a mixed impedance ground has been examined. The time-frequency decomposition does not present any interference pattern over the frequency band of interest. The gap plays an important role when the source is in front of the receiver, but its effect on the acoustic field is negligible when the distance source-receiver is large. In both configurations, the Doppler shift is exhibited.

Time-domain simulations of outdoor sound propagation are now mature for applications in industry. As shown in this paper, they allow one to consider complex environments, with a topography and a mixed impedance ground, and can account for any source trajectory. Time signals obtained directly from the results of the simulations without any further assumption can be used for auralization. In near future, they can be applied for virtual certification of acoustic performance under the condition that transportation noise sources are sufficiently well described.

\section{ACKNOWLEDGEMENTS}

This work was performed within the framework of the Labex CeLyA of Universite de Lyon, operated by the French National Research Agency (ANR-10-LABX-0060/ANR11-IDEX-0007). It was granted access to the HPC resources of IDRIS under the allocation 2014-022203 made by GENCI (Grand Equipement National de Calcul Intensif ).

\section{REFERENCES}

[1] European environment agency, "A closer look at urban transport. TERM 2013: transport indicators tracking progress towards environmental targets in Europe", 2013, pp. 1-106.

[2] Norum, T. D. and Liu, C. H., "Point source moving above a finite impedance reflecting plane-experiment and theory", J. Acoust. Soc. Am., Vol. 63, No. 4, 1978, pp. 1069-1073.

[3] Oie, S. and Takeuchi, R., "Sound radiation from a point source moving in parallel to a plane surface porous material", Acustica, Vol. 48, No. 3, 1981, pp. 123-129. 
[4] Attenborough, K., Li, K. M. and Horoshenkov, K., Predicting outdoor sound, Taylor \& Francis, 2007.

[5] Ochmann, M., "Exact solutions for sound radiation from a moving monopole above an impedance plane", J. Acoust. Soc. Am., Vol. 133, No. 4, 2013, pp. 1911-1921.

[6] Bongini, E., Molla, S., Gautier, P. E., Habault, D., Mattei, P. O. and Poisson, F., "Synthesis of noise of operating vehicles: development within SILENCE of a tool with listening features", Noise and Vibration Mitigation for Rail Transportation Systems, 2008, pp. 320-326.

[7] Arntzen, M. and Simons, D. G., "Modeling and synthesis of aircraft flyover noise”, Appl. Acoust., Vol. 84, 2014, pp. 99-106.

[8] Cotté, B., Blanc-Benon, P., Cremezi-Charlet, C. and Poisson, F., "High-speed train noise propagation at mid-ranges", Nineteenth ICA, Madrid, Spain, 2007.

[9] Van Renterghem, T. and Botteldooren, D., "Numerical simulation of the effect of trees on downwind noise barrier performance", Acta Acust. united Ac., Vol. 89, 2003, pp. 764-778.

[10] Ostashev, V. E., Wilson, D. K., Liu, L., Aldridge, D. F., Symons, N. P. and Marlin, D., "Equations for finite-difference, time-domain simulation of sound propagation in moving inhomogeneous media and numerical implementation", J. Acoust. Soc. Am., Vol. 117, 2005, pp. 503-517.

[11] Hornikx, M., Waxler, R. and Forssén, J., "The extended Fourier pseudospectral time-domain method for atmospheric sound propagation", J. Acous. Soc. Am. I Vol. 128, 2010, pp. 1632-1646.

[12] Dragna, D., Blanc-Benon, P. and Poisson, F., "Time-domain solver in curvilinear coordinates for outdoor sound propagation over complex terrain", J. Acoust. Soc. Am. Vol. 133, No. 6, 2013, pp. 3751-3763.

[13] Guillaume, G., Aumond, P., Gauvreau, B. and Dutilleux, G., "Application of the transmission line matrix method for outdoor sound propagation modelling-Part 1: Model presentation and evaluation", Appl. Acoust., Vol. 76, 2014, pp. 113-118.

[14] Aumond, P., Guillaume, G., Gauvreau, B., Lac, C., Masson, V. and Bérengier, M., "Application of the Transmission Line Matrix method for outdoor sound propagation modelling - Part 2: Experimental validation using meteorological data derived from the meso-scale model Meso-NH", Appl. Acoust., Vol. 76, 2014, pp. 107-112.

[15] Marsden, O., Bogey, C. and Bailly, C., "High-order curvilinear simulations of flows around non-Cartesian bodies", J. Comp. Acoust., Vol. 13, 2005, pp. 731-748.

[16] Tam, C. K. W. and Webb, J. C., "Dispersion-relation preserving finite difference schemes for computational acoustics", J. Comp. Phys., Vol. 107, 1993, pp. 262-281.

[17] Bogey, C. and Bailly, C., "A family of low dispersive and low dissipative explicit schemes for flow and noise computations", J. Comp. Phys., Vol. 194, 2004, pp. 194-214.

[18] Berland, J., Bogey, C., Marsden, O. and Bailly, C., "High order, low dispersive and low dissipative explicit schemes for multiple-scale and boundary problems", J. Comp. Phys., Vol. 224, 2002, pp. 637-662. 
[19] Fornberg, B., "The pseudospectral method: comparisons with finite-differences for the elastic wave equation", Geophysics., Vol. 52, No. 4, 1987, pp. 483-501.

[20] Hu, F. Q., Hussaini, M. Y. and Manthey, J. L., "Low-dissipation and lowdispersion Runge-Kutta schemes for computational acoustics", J. Comp. Phys. Vol. 124, 1996, pp. 177-191.

[21] Berland, J., Bogey, C. and Bailly, C., "Low-dissipation and low-dispersion fourthorder Runge-Kutta algorithm”, Computers \& Fluids, Vol. 35, 2006, pp. 1459-1463.

[22] Boyd, J. P., Chebyshev and Fourier spectral methods, Dover Publications, 2000.

[23] Huang, X. and Zhang, X., "A Fourier pseudospectral method for some computational aeroacoustics problems", Int. J. Aeroacoustics Vol. 5, No. 3, 2006, pp. 279-294.

[24] Bogey, C., de Cacqueray, N. and Bailly, C., "A shock-capturing methodology based on adaptative spatial filtering for high-order non-linear computations", $J$. Comp. Phys. Vol. 228, 2009, pp. 1447-1465.

[25] Rienstra, S. W., "Impedance models in time domain including the Helmholtz resonator model", Twelfth AIAA/CEAS Aeroacoustics Conference, Cambridge, MA, USA, 2006, AIAA Paper 2006-2686.

[26] Dragna, D. and Blanc-Benon, P., "Physically admissible impedance models for time-domain computations of outdoor sound propagation", Acta Acust. united Ac., Vol. 100, 2014, pp. 401-410.

[27] Delany, M. and Bazley, E., “Acoustical properties of fibrous absorbent materials”, Appl. Acoust., Vol. 3, 1970, pp. 105-116.

[28] Luebbers, R. J. and Hunsberger, F., "FDTD for Nth-order dispersive media", IEEE Trans. Antennas Propag., Vol. 40, 1992, pp. 1297-1301.

[29] Reymen, Y., Baelmans, M. and Desmet, W., "Time-Domain Impedance Formulation based on Recursive Convolution", Twelfth AIAA/CEAS Aeroacoustics Conference, Cambridge, MA, USA, 2006, AIAA Paper 2006-2685.

[30] Cotté, B., Blanc-Benon, P., Bogey C. and Poisson, F., "Time-domain impedance boundary conditions for simulations of outdoor sound propagation", AIAA J., Vol. 47, No. 10, 2009, pp. 2391-2403.

[31] Gustavsen, B., Semlyen, A., "Rational approximation of frequency domain responses by vector fitting", IEEE Trans. Power Delivery, Vol. 14, No. 3, 1999, pp. 1052-1061.

[32] Miki, Y., "Acoustical properties of porous materials-Modifications of DelanyBazley models", J. Acoust. Soc. Jpn., Vol. 11, No. 1, 1990, pp. 19-24.

[33] Salomons, E., Blumrich, R. and Heimann, D., "Eulerian time-domain model for sound propagation over a finite-impedance ground surface. Comparison with frequency-domain models", Acta Acust. united Ac., Vol. 88, 2002, pp. 483-492.

[34] Bérenger, J. P., "A perfectly matched layer for the absorption of electromagnetic waves”, J. Comp. Phys. Vol. 114, 1994, pp. 185-200. 
[35] Habault, D. and Filippi, P. J. T., "Ground effect analysis: surface wave and layer potential representations”, J. Sound. Vib., Vol. 79, No. 4, 1981, pp. 529-550.

[36] Dragna, D., Cotté, B., Blanc-Benon, P. and Poisson, F., "Time-domain simulations of outdoor sound propagation with suitable impedance boundary conditions", AIAA J., Vol. 49, No. 7, 2011, pp. 1420-1428.

[37] Bogey, C. and Bailly, C., "Three-dimensional non-reflective boundary conditions for acoustic simulations: far-field formulation and validation test cases", Acta Acust. united Ac., Vol. 88, 2002, pp. 463-471.

[38] Mohseni, K. and Colonius, T., "Numerical treatment of polar coordinate singularities", J. Comp. Phys., Vol. 157, 2000, pp. 787-795.

[39] Bogey, C., de Cacqueray, N. and Bailly, C., "Finite differences for coarse azimuthal discretization and for reduction of effective resolution near origin of cylindrical flow equations", J. Comp. Phys., Vol. 230, 2011, pp. 1134-1146.

[40] Dragna, D., Blanc-Benon, P. and Poisson, F., "Modeling of broadband moving sources for time-domain simulations of outdoor sound propagation", AIAA J., Vol. 52, No. 9, 2014, pp. 1928-1939.

[41] Roger, M., "Sound radiation by moving surfaces and the Green's functions technique theoretical acoustics", Noise sources in turbulent shear flows: fundamentals and applications, edited by R. Camussi, Springer, 2013, pp. 73-116.

[42] Dragna, D., Blanc-Benon, P. and Poisson, F., "Impulse propagation over a complex site: A comparison of experimental results and numerical predictions", J. Acoust. Soc. Am., Vol. 135, No. 3, 2014, pp. 1096-1105.

[43] Bérengier, M., Stinson, M. R., Daigle, G. A. and Hamet, J. F., "Porous road pavement: acoustical characterization and propagation effects", J. Acoust. Soc. Am. Vol. 101, No. 1, 1997, pp. 155-162. 